\title{
Secondary sentinel lymph node tracing technique: a new method for tracing lymph nodes in radical gastrectomy for advanced gastric cancer"
}

\author{
Zong-lin $\mathrm{LI}^{\dagger 1}$, Huai-wu JIANG ${ }^{2}$, Min SONG ${ }^{3}$, Liang XU' ${ }^{1}$, Dong XIA ${ }^{1}$, Qing LIU ${ }^{1}$ \\ $\left({ }^{1}\right.$ Department of Gastrointestinal Surgery, the First Affiliated Hospital of Sichuan Medical University, Luzhou 646000, China) \\ ( ${ }^{2}$ Department of Gastrointestinal Surgery, the Second Affiliated Hospital of North Sichuan Medical College, Mianyang 621000, China) \\ ( ${ }^{3}$ Department of Laboratory Medicine, the First Affiliated Hospital of Sichuan Medical University, Luzhou 646000, China) \\ †E-mail: lizonglin85@163.com
}

Received Mar. 7, 2015; Revision accepted Aug. 4, 2015; Crosschecked Oct. 21, 2015

\begin{abstract}
Objective: To explore the feasibility and clinical value of secondary sentinel lymph node (SSLN) tracing technique in radical gastrectomy for advanced gastric cancer (AGC). Methods: From January 2009 to June 2011, 247 patients who suffered from gastric angle cancer with metastasis in No. 3 group lymph nodes were divided randomly into groups $A$ and $B$. Methylthioninium chloride was injected into the peripheral tissue of the metastatic No. 3 group lymph nodes of 138 patients in group A before tumor resections. SSLNs were traced and individual lymphadenectomies were carried out based on the biopsy results of the SSLNs. Standard D2 radical gastrectomies were carried out directly on 109 patients in group B. Postoperative follow-up and survival analysis were carried out for patients in both groups. Results: SSLNs were found in 114 (82.6\%) patients in group A. Ninety of those patients (78.9\%) demonstrated existing metastasis in SSLNs. According to Kaplan-Meier's method, the postoperative 3-year cumulative survival rates were $63.5 \%$ and $47.5 \%$, and the median survival time were 40 and 36 months for the patients of groups $A$ and $B$, respectively $(P<0.05)$. Conclusions: The SSLN tracing technique is feasible in radical gastrectomy for AGC. It gives surgeons important information about the terminal status of lymph node metastasis and provides some scientific basis for individual lymphadenectomy.
\end{abstract}

Key words: Secondary sentinel lymph node (SSLN), Advanced gastric cancer (AGC), Individual lymphadenectomy, Survival analysis

doi: 10.1631 jzus.B1500053

Document code: A

CLC number: R735.2

\section{Introduction}

Sentinel lymph nodes (SLNs) refer to those lymph nodes which are the first to directly receive the lymphatic drainage of primary tumors and which are most likely to contain metastases. SLNs can be more than one lymph node and also can exist in more than one direction (Fig. 1) (Chen et al., 2006; Tangoku

\footnotetext{
* Project supported by the Projects of Sichuan Provincial Health Department Scientific Research (Nos. 050182 and 090250), China (D) ORCID: Zong-lin LI, http://orcid.org/0000-0002-5240-9858 (C) Zhejiang University and Springer-Verlag Berlin Heidelberg 2015
}

et al., 2007). SLN tracing techniques have been widely used in clinical practice for many solid tumors such as melanoma, breast cancer, and colorectal carcinoma, and excellent clinical results have been achieved (Shimazu and Noguchi, 2011; Erman et al., 2012; van der Zaag et al., 2012). Some preliminary research on SLN tracing techniques has been carried out since the concept of SLN was introduced to the area of gastric cancer in the 1990s. The techniques have generally been found to be valuable in the treatment of early gastric cancer (EGC) but of little or no value in the treatment of advanced gastric cancer (AGC). This is because, in AGC, real SLNs could not 
be found by routine SLN tracing techniques (Dong et al., 2012; Tóth et al., 2012; Chen et al., 2014; Symeonidis et al., 2014). Therefore, exploring the detection methods and assessing the value of SLN tracing techniques in $\mathrm{AGC}$ is of great significance. Based on the accumulated knowledge, we proposed the concept of secondary sentinel lymph nodes (SSLNs). SSLNs refer to those SLNs which are identified according to the basic methods for detecting SLN, and by injecting tracer into the peripheral tissue of metastatic lymph nodes to trace lymph nodes which are beyond those metastatic lymph nodes (Fig. 2). We used the SSLN tracing technique to research AGC, aiming at providing some scientific basis for individual lymphadenectomy in radical gastrectomy of AGC.

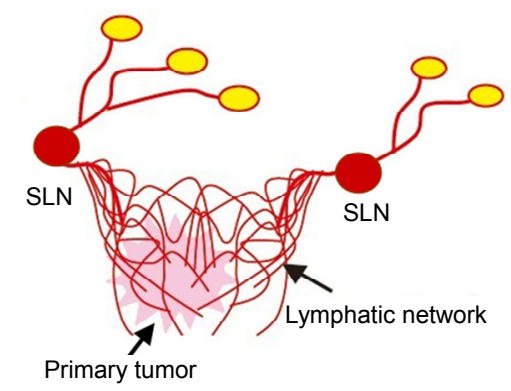

Fig. 1 Schematic diagram of sentinel lymph node (SLN)

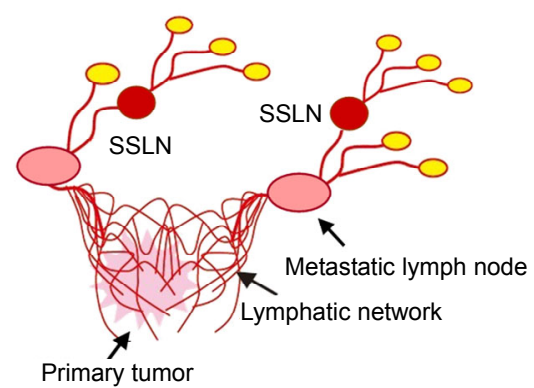

Fig. 2 Schematic diagram of secondary sentinel lymph node (SSLN)

\section{Patients and methods}

\subsection{Patients}

From January 2009 to June 2011, 247 patients were enrolled in the study, consisting of 135 males and 112 females, aged from 38 to 67 years old and with a median age of 52 years. They were treated at the First Affiliated Hospital of Sichuan Medical
University (Luzhou, China). All of these patients were suffering from gastric angle cancer accompanied by metastatic nodule formation of the No. 3 group lymph nodes. The identifying criteria were: (1) No. 3 group lymph nodes were found during surgery to be enlarged, hard, and accompanied by cancerous nodule formation or fusion; (2) No. 3 group lymph nodes were found to contain cancer cells by postoperative pathological examination. No metastatic nodule formation was found in other groups of lymph nodes. The primary tumors of these patients were localized, without hepatic metastases and other distant metastases, according to the results of preoperative computed tomography, and no patients received neoadjuvant chemoradiotherapy, interventional therapy, or other special treatments. The diameter of the primary tumor was less than $5 \mathrm{~cm}$ in 156 cases and greater than or equal to $5 \mathrm{~cm}$ in 91 cases. All the patients belonged to the T3 or T4 stage according to tumor node metastasis (TNM) staging and there were 130 cases in which the tumors were well differentiated or moderately differentiated adenocarcinomas, 84 cases in which the tumors were poorly differentiated or undifferentiated adenocarcinomas, and 33 cases in which the tumors were mucinous or signet ring cell carcinomas.

\subsection{Research methods}

The 247 AGC patients were divided into groups $\mathrm{A}$ and $\mathrm{B}$ randomly. Two milliliter methylthioninium chloride was injected into the $3,6,9$, and 12 point positions of the peripheral tissue of the metastatic No. 3 group lymph nodes of each of the 138 patients in group A before tumor resections. Tracer material could be seen extending some distance along the lymphatic vessels. SSLNs were defined as the stained lymph nodes nearest to the metastatic No. 3 group lymph nodes that were found within 5 to $10 \mathrm{~min}$ after injecting methylthioninium chloride (Fig. 3). The number and distribution of SSLNs were observed and recorded. These SSLNs were resected and examined pathologically to determine whether they contained metastases. If SSLNs were positive, D2 lymphadenectomy plus regional lymph node dissection was selected. If SSLNs were negative, only D2 lymphadenectomy was carried out. If SSLNs were not found, D2 lymphadenectomy was carried out for the patients in group A, but only standard D2 radical 
gastrectomy was carried out for the 109 patients in group B. Postoperative tumor tissue and lymph nodes were subjected to hematoxylin-eosin (HE) and immunohistochemistry (IHC) staining for further study. The patients whose postoperative survival time exceeded 24 months received 8 cycles of adjuvant chemotherapy at $1,2,3,6,9,12,18$, and 24 months after surgery. The chemotherapy regimen was tegafur injection ( 1 g every other day (q.o.d.)) plus topotecan hydrochloride injection ( $2 \mathrm{mg}$ q.o.d.) and these patients received chemotherapy for $5 \mathrm{~d}$ during each cycle. Patients whose postoperative survival time did not exceed 24 months received adjuvant chemotherapy

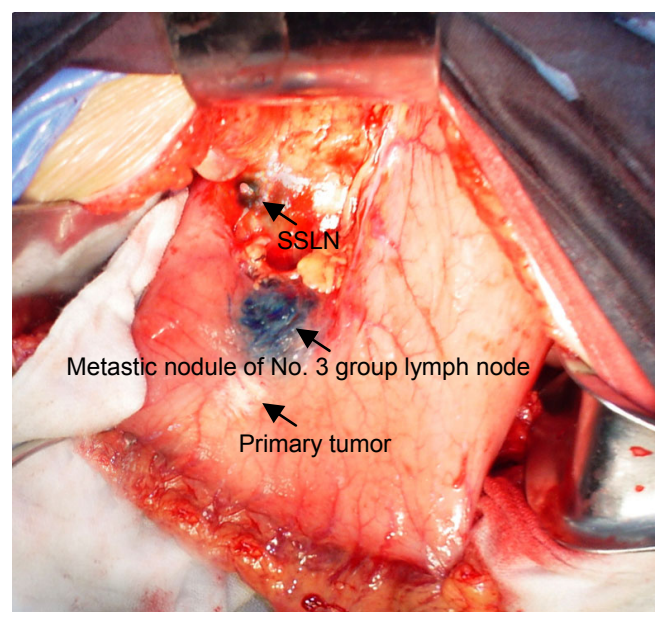

Fig. 3 Secondary sentinel lymph node (SSLN) tracing during operation of corresponding cycles before death. Postoperative follow-up and survival analysis were carried out for patients in both groups. The postoperative follow-up period was from 4 to 40 months.

\subsection{Statistical analysis}

Data were analyzed using SPSS 17.0 statistical software and count data were analyzed by chi-square tests. Cumulative survival rates were calculated by Kaplan-Meier's method and the differences were evaluated by log-rank test. A $P$-value of $<0.05$ was considered to indicate statistical significance.

\section{Results}

\subsection{Analysis of differences in overall composition between groups $A$ and $B$}

There were no significant differences in sex, age, tumor size, pathomorphism, degree of differentiation, or staging of the tumors between the two groups of patients (Table 1).

\subsection{SSLN tracing for the patients in group A}

SSLNs were found in $114(82.6 \%)$ patients in group A. Among those patients, 71 were found by intra-operative HE staining to have metastasis in SSLNs and 19 were found by postoperative IHC staining to have micrometastasis in SSLNs. Thus, a

Table 1 Comparison of the overall composition between groups A and B

\begin{tabular}{|c|c|c|c|c|}
\hline \multirow{2}{*}{ Parameter } & \multicolumn{2}{|c|}{ Number of patients } & \multirow{2}{*}{$\chi^{2}$-value } & \multirow{2}{*}{$P$-value } \\
\hline & Group A & Group B & & \\
\hline Gender & & & 0.390 & 0.532 \\
\hline Male & 73 & 62 & & \\
\hline Female & 65 & 47 & & \\
\hline Age & & & 0.564 & 0.452 \\
\hline$<50$ years & 61 & 43 & & \\
\hline$\geq 50$ years & 77 & 66 & & \\
\hline Diameter of tumor & & & 0.002 & 0.967 \\
\hline$<5 \mathrm{~cm}$ & 87 & 69 & & \\
\hline$\geq 5 \mathrm{~cm}$ & 51 & 40 & & \\
\hline Pathomorphism & & & 0.489 & 0.484 \\
\hline Ulcerative type & 92 & 68 & & \\
\hline Protruding type & 46 & 41 & & \\
\hline Degree of differentiation & & & 0.379 & 0.828 \\
\hline Well or moderately differentiated adenocarcinoma & 75 & 55 & & \\
\hline Poorly or undifferentiated adenocarcinoma & 45 & 39 & & \\
\hline Mucinous adenocarcinoma and signet ring cell carcinoma & 18 & 15 & & \\
\hline Staging of tumor & & & 0.562 & 0.454 \\
\hline $\mathrm{T} 3$ & 53 & 47 & & \\
\hline $\mathrm{T} 4$ & 85 & 62 & & \\
\hline
\end{tabular}


total of 90 patients were shown to have metastasis in SSLNs by HE and IHC staining, with a positive rate of $78.9 \%$ (90/114). A total of 204 SSLNs were found, of which 156 were shown to have existing metastasis. The number of SSLNs detected, the number of positive SSLNs, the percentage of positive SSLNs in each lymph node group, and the extent of lymphadenectomy for those patients whose SSLNs were positive are shown in Table 2. The rate of positive SSLNs was higher for those patients whose primary tumor diameter were greater than or equal to $5 \mathrm{~cm}$ and whose tumors were poorly differentiated. According to chi-square tests, there were significant differences between groups $(P<0.05$; Table 3$)$.

\subsection{Analysis of perioperative complications for the patients in groups $A$ and $B$}

Several patients suffered from postoperative anastomotic leakage, intra-abdominal hemorrhage, gastrointestinal tract obstruction, biliary fistula, pancreatic fistula, and gastroplegia in both groups A and B. However, there were no significant differences in these complications between groups $\mathrm{A}$ and $\mathrm{B}$ according to chi-square tests $(P>0.05$; Table 4$)$.

\subsection{Survival analysis for the patients in groups $\mathrm{A}$ and $B$}

The postoperative 1-, 2-, and 3-year cumulative survival rates were $95.7 \%, 89.8 \%$, and $63.5 \%$, respectively, for patients in group A, and $89.9 \%, 72.4 \%$, and $47.5 \%$, respectively, for patients in group $B$. The median survival time was 40 months for group A and 36 months for group B. According to log-rank tests, there were significant differences between groups $(P<0.05)$. The survival curves are shown in Fig. 4.

Table 2 Number of SSLNs detected, number of positive SSLNs, the rate of positive SSLNs in each lymph node group, and the extent of lymphadenectomy for those patients whose SSLNs were positive for the patients in group A

\begin{tabular}{ccccc}
\hline $\begin{array}{c}\text { Lymph node } \\
\text { group }\end{array}$ & $\begin{array}{c}\text { Number of } \\
\text { SSLNs detected }\end{array}$ & $\begin{array}{c}\text { Number of } \\
\text { positive SSLNs }\end{array}$ & $\begin{array}{c}\text { Rate of positive } \\
\text { SSLNs (\%) }\end{array}$ & $\begin{array}{c}\text { Extent of lymphadenectomy for those } \\
\text { patients whose SSLNs were positive }\end{array}$ \\
\hline No. 5 & 48 & 42 & 87.5 & Nos. 1, 3-9, 11-13 \\
No. 7 & 120 & 93 & 77.5 & Nos. 1-11 \\
No. 8 & 24 & 15 & 62.5 & Nos. 1, 3-9, 11-13 \\
No. 9 & 12 & 6 & 50.0 & Nos. 1, 3-9, 11-13, 16 \\
\hline
\end{tabular}

Table 3 Relationship between SSLNs and clinical pathological features of AGC for the patients in group A

\begin{tabular}{lcccr}
\hline \multicolumn{1}{c}{ Clinical pathological features of AGC } & $\begin{array}{c}\text { Number of } \\
\text { SSLNs detected }\end{array}$ & $\begin{array}{c}\text { Number of } \\
\text { positive SSLNs }\end{array}$ & $\begin{array}{c}\text { Rate of positive } \\
\text { SSLNs (\%) }\end{array}$ & $\chi^{2}$-value $P$-value \\
\hline $\begin{array}{l}\text { Diameter of primary tumor } \\
<5 \mathrm{~cm}\end{array}$ & 75 & 54 & 72.0 & 6.366 \\
$\quad \geq 5 \mathrm{~cm}$ & 39 & 36 & 92.3 & \\
$\quad \begin{array}{l}\text { Degree of tumor's differentiation } \\
\text { Well or moderately differentiated } \\
\quad \text { adenocarcinoma }\end{array}$ & 59 & 42 & 71.2 & \\
$\begin{array}{l}\text { Poorly or undifferentiated adenocarcinoma, } \\
\text { mucinous adenocarcinoma, and }\end{array}$ & 55 & 48 & 87.3 & 0.035 \\
$\quad$ signet ring cell carcinoma & & & & \\
\hline
\end{tabular}

Table 4 Analysis of perioperative complications for patients in groups A and B

\begin{tabular}{ccccccccc}
\hline \multirow{2}{*}{ Group } & Case & \multicolumn{7}{c}{ Case of perioperative complications } \\
\cline { 3 - 9 } & & $\begin{array}{c}\text { Anastomotic } \\
\text { leakage }\end{array}$ & $\begin{array}{c}\text { Intra-abdominal } \\
\text { hemorrhage }\end{array}$ & $\begin{array}{c}\text { Gastrointestinal } \\
\text { tract obstruction }\end{array}$ & $\begin{array}{c}\text { Biliary } \\
\text { fistula }\end{array}$ & $\begin{array}{c}\text { Pancreatic } \\
\text { fistula }\end{array}$ & Gastroplegia & Death \\
\hline A & 138 & 2 & 2 & 3 & 1 & 2 & 1 & 0 \\
B & 109 & 3 & 1 & 2 & 0 & 1 & 0 & 0 \\
\hline
\end{tabular}




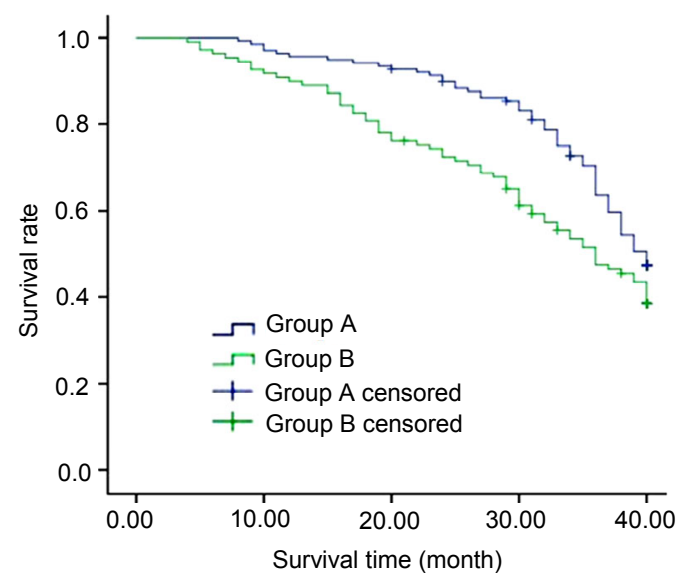

Fig. 4 Survival curves of groups $A$ and B

\section{Discussion}

\subsection{Characteristics of SSLN tracing for advanced gastric angle cancer}

The detection rate and the rate of positive SLNs reported by Wang et al. (2012) were $93.7 \%$ and $76.9 \%$, respectively, but in this study the corresponding rates for SSLNs were $82.6 \%$ and $78.9 \%$ for the patients in group A. Clearly, the detection rate of SSLNs was lower than that reported for SLNs. The reason for this result may be that the normal lymphatic drainage system had been damaged by cancer cells in AGC. However, the number of SSLNs detected was higher in the No. 7 group than in any other groups. In addition, the rate of positive SSLNs was higher in the No. 5 and No. 7 groups but lower in the No. 8 and No. 9 groups. This phenomenon was in line with the general rule relating to the location of the lymph node metastasis. The rate of positive SSLNs was higher in patients whose primary tumor diameter was greater than or equal to $5 \mathrm{~cm}$ and whose tumors were poorly differentiated. This phenomenon was consistent with the pathological features of AGC, which indicate that larger tumors are more differentiated and have a greater likelihood of lymph node metastasis, more lymph node metastases, and a worse prognosis for the patients.

In this study, 19 patients in group A were shown by IHC staining to have metastases in SSLNs which were not revealed by HE staining. This result suggests that the false negative rate is higher using conventional HE staining, which is consistent with the results of Miyashiro et al. (2014). Micrometastasis is not only an adverse prognostic factor, but also a major cause of tumor recurrence. Therefore, sensitive and accurate detection methods, such as IHC or reverse transcription polymerase chain reaction (RT-PCR), should be used to detect micrometastasis of SSLNs in AGC. Studies have reported that rapid, sensitive IHC and RT-PCR can detect micrometastases in a few minutes (Lee et al., 2012; Hirayama et al., 2014). If true, then the value of the SSLN tracing technique for individual lymphadenectomy of AGC will be more significant.

\subsection{Basic theory and methods of the SSLN tracing technique}

The SSLN tracing technique is based on the premise that not every group of lymph nodes is invaded by metastatic carcinoma cells in AGC, and that large-scale removal of innocuous tissue should be avoided. Also, attention should be paid to abnormal lymphatic drainage as well as jumping lymph node metastasis. Most importantly, metastatic lymph nodes must not be missed during radical gastrectomy for AGC. However, real SLNs cannot be found by conventional SLN tracing techniques in AGC, especially in the T3 and T4 stages of AGC. Because most primary tumors are relatively large, parts of lymph vessels can be obstructed by tumor cells and normal lymphatic drainage damaged. Therefore, lymph tracer must be injected into surrounding metastases of primary tumors in those AGC cases in which metastatic nodules are present in order to understand the terminal status of lymph node metastasis and provide some scientific basis for individual lymphadenectomy of AGC at different stages.

The basic methods of the SSLN tracing technique include the following: in radical gastrectomy of AGC, lymph tracer must be injected into the peripheral tissue of metastases surrounding the primary tumor. SSLNs are the stained lymph nodes nearest to metastases surrounding the primary tumor that are found within 5 to $10 \mathrm{~min}$ after injecting lymph tracer. SSLNs are resected and pathological examination carried out quickly. If there are cancerometastases in SSLNs, D2 lymphadenectomy plus regional lymph node dissection of positive SSLNs will be performed. If there are no cancerometastases in SSLNs, only D2 lymphadenectomy is performed. The regional lymph nodes of negative SSLNs must be retained. 
Note that whether EGC or AGC, if there are no metastatic nodules around the primary tumor, the conventional SLN tracing technique should be selected. If there are metastatic nodules around the primary tumor, then the SSLN tracing technique should be selected.

\subsection{Clinical value of the SSLN tracing technique in radical gastrectomy for AGC}

Among the most common tumors, gastric cancers are found in modern times all over the world. Although the detection rate of EGC has been increasing in recent years, AGC continues to account for most gastric cancers. Because of the intricacy of lymphatic drainage and the presence of skip metastases, part of the lymphatic drainage can be blocked by tumor cells in AGC, and there is a high false negative rate for SLNs. Therefore, most clinicians believe that SLN tracing techniques are of little value in AGC. Studies have shown that the positive rate of SLN in gastric cancer decreases as the depth of invasion by the tumor increases. The positive rates of SLN were $100 \%, 91.6 \%$, and $62.5 \%$ in the $\mathrm{T} 1, \mathrm{~T} 2$, and $\mathrm{T} 3$ stages, respectively. So SLNs provide little surgical guidance for gastric cancer in the $\mathrm{T} 3$ or $\mathrm{T} 4$ stages (Mochiki et al., 2006). Therefore, how to improve the value of SLN tracing techniques in guiding radical gastrectomy for AGC is an important and urgent research issue. Fortunately, SSLN tracing techniques can provide some scientific basis for individual lymphadenectomy of AGC. In this study, several patients in both groups suffered from postoperative anastomotic leakage, intra-abdominal hemorrhage, gastrointestinal tract obstruction, biliary fistula, pancreatic fistula and gastroplegia, but there were no significant differences in these complications between the groups $(P>0.05)$. This suggests that carrying out extended radical gastrectomy did not increase the surgical risk nor negatively impact the postoperative recovery of patients with AGC. According to survival analysis, the postoperative 1-, 2-, and 3-year cumulative survival rates and the median survival time for the patients of group A were significantly higher than those for the patients of group $\mathrm{B}(P<0.05)$, which suggested that the SSLN tracing technique is feasible in radical gastrectomy for AGC and individual lymphadenectomy is important in improving the prognosis of AGC. Currently, Japanese guidelines for the treatment of AGC are to perform D2 lymphadenectomy (Japanese Gastric Cancer Association, 2011). We approve of the recommendation of D2 lymphadenectomy as the standard operation for AGC and do not recommend $\mathrm{D} 2^{+}$or $\mathrm{D} 3$ lymphadenectomy when there is no scientific guiding ideology. However, if there is scientific guiding ideology such as the theory and technology of SSLNs, we believe that extended lymphadenectomy is of significance for AGC.

Gastric angle cancer is a common clinical type of gastric cancer. No. 3 group lymph nodes are often the first metastatic lymph nodes, so we selected the metastatic No. 3 group lymph nodes as the starting point for SSLN tracing in this study. However, different starting points of SSLN tracing should be selected for AGC of different parts in clinical studies. For example, No. 5 or No. 6 group lymph nodes can be selected as starting points for SSLN tracing for gastric antrum cancer. In addition, multiple metastases surrounding the primary tumor should be selected as starting points for SSLN tracing so that clinicians can fully understand the terminal status of lymph node metastasis for AGC. In summary, the condition of SSLNs can accurately predict the metastatic condition of regional lymph nodes for AGC patients. According to the biopsy results from SSLNs, unnecessary extended radical resection can be avoided. Furthermore, jumping metastatic lymph nodes, abnormal lymphatic drainage and micrometastasis can be discovered by SSLN tracing so that the failure to detect metastatic lymph nodes can be prevented. As a result, the recurrence rate of tumors will be reduced and the survival rate of patients will be improved. The theory and tracing technique for SSLN in AGC have not only enriched the theoretical understanding of the SLN system and expanded the range of application of SLN tracing techniques, but also provided a new method and some scientific basis for individual lymphadenectomy of AGC at different stages.

\section{Compliance with ethics guidelines}

Zong-lin LI, Huai-wu JIANG, Min SONG, Liang XU, Dong XIA, and Qing LIU declare that they have no conflict of interest.

All procedures followed were in accordance with the ethical standards of the responsible committee on human experimentation (institutional and national) and with the Helsinki Declaration of 1975, as revised in 2008 (5). Informed 
consent was obtained from all patients for being included in the study.

\section{References}

Chen, R., He, Q., Cui, J., et al., 2014. Lymph node metastasis in early gastric cancer. Chin. Med. J., 127(3):560-567. [doi:10.3760/cma.j.issn.0366-6999.20123235]

Chen, S.L., Iddings, D.M., Scheri, R.P., et al., 2006. Lymphatic mapping and sentinel node analysis: current concepts and applications. CA Cancer J. Clin., 56(5):292-309. [doi:10.3322/canjclin.56.5.292]

Dong, L.F., Wang, L.B., Shen, J.G., et al., 2012. Sentinel lymph node biopsy predicts lymph node metastasis in early gastric cancer: a retrospective analysis. Dig. Surg., 29(2):124-129. [doi:10.1159/000336210]

Erman, A.B., Collar, R.M., Griffith, K.A., et al., 2012. Sentinel lymph node biopsy is accurate and prognostic in head and neck melanoma. Cancer, 118(4):1040-1047. [doi:10. 1002/cncr.26288]

Hirayama, R., Seshimo, A., Miyake, K., et al., 2014. Intraoperative diagnosis of lymph node metastasis by transcription-reverse transcription concerted reaction assay in gastric cancer. Int. J. Clin. Oncol., 19(3):473-478. [doi:10.1007/s10147-013-0579-9]

Japanese Gastric Cancer Association, 2011. Japanese gastric cancer treatment guidelines 2010 (ver. 3). Gastric Cancer, 14(2):113-123. [doi:10.1007/s10120-011-0042-4]

Lee, H.S., Lee, H.E., Park, D.J., et al., 2012. Precise pathologic examination decreases the false-negative rate of sentinel lymph node biopsy in gastric cancer. Ann. Surg. Oncol., 19(3):772-778. [doi:10.1245/s10434-011-2106-4]

Miyashiro, I., Hiratsuka, M., Sasako, M., et al., 2014. High false-negative proportion of intraoperative histological examination as a serious problem for clinical application of sentinel node biopsy for early gastric cancer: final results of the Japan Clinical Oncology Group multicenter trial JCOG0302. Gastric Cancer, 17(2):316-323. [doi:10. 1007/s10120-013-0285-3]

Mochiki, E., Kuwano, H., Kamiyama, Y., et al., 2006. Sentinel lymph node mapping with technetium-99m colloidal rhenium sulfide in patients with gastric carcinoma. Am. J. Surg., 191(4):465-469. [doi:10.1016/j.amjsurg.2005.09. 002]

Shimazu, K., Noguchi, S., 2011. Sentinel lymph node biopsy before versus after neoadjuvant chemotherapy for breast cancer. Surg. Today, 41(3):311-316. [doi:10.1007/s00595010-4404-z]

Symeonidis, D., Koukoulis, G., Tepetes, K., 2014. Sentinel node navigation surgery in gastric cancer: current status. World J. Gastrointest. Surg., 6(6):88-93. [doi:10.4240/ wjgs.v6.i6.88]

Tangoku, A., Seike, J., Nakano, K., et al., 2007. Current status of sentinel lymph node navigation surgery in breast and gastrointestinal tract. J. Med. Invest., 54(1-2):1-18. [doi:10.2152/jmi.54.1]

Tóth, D., Kathy, S., Csoban, T., et al., 2012. Prospective comparative study of sentinel lymph node mapping in gastric cancer-submucosal versus subserosal marking method. Magy. Seb., 65(1):3-8. [doi:10.1556/MaSeb.65. 2012.1.1]

van der Zaag, E.S., Bouma, W.H., Peters, H.M., et al., 2012. Implications of sentinel lymph node mapping on nodal staging and prognosis in colorectal cancer. Colorectal Dis., 14(6):684-690. [doi:10.1111/j.1463-1318.2012.029 49.x]

Wang, Z., Dong, Z.Y., Chen, J.Q., et al., 2012. Diagnostic value of sentinel lymph node biopsy in gastric cancer: a meta-analysis. Ann. Surg. Oncol., 19(5):1541-1550. [doi:10.1245/s10434-011-2124-2]

\section{中文概要}

题 目: 二级前哨淋巴结示踪技术: 进展期胃癌根治术中 淋巴结示踪新方法

目 的: 探讨二级前哨淋巴结 (SSLN) 示踪技术在进展 期胃癌（AGC）根治术中的可行性及临床价值。

创新点: 提出 SSLN 概念并以 SSLN 理论为指导对 AGC 实施个体化淋巴结清扫术。

方 法: 将 2009 年 1 月至 2011 年 6 月于我院手术治疗的 进展期胃角部癌患者 247 例随机分为 A、B 两组: A 组 138 例于术中肿瘤切除前向第 3 组淋巴结转 移结节边缘注亚甲蓝示踪剂, 寻找并切取 SSLN 进行病理活检, 并根据 SSLN 的活检结果进行个 体化淋巴结清扫术; B 组 109 例直接进行标准 D2 胃癌根治术。对 A、B 组患者进行术后随访和生 存分析。

结 论: SSLN 示踪技术应用于 AGC 手术是可行的。 SSLN 示踪技术可以了解 AGC 淋巴结转移的终末 状态, 对指导 AGC 个体化淋巴结清扫及改善预 后具有重要的临床价值。

关键词: 二级前哨淋巴结; 进展期胃癌; 个体化淋巴结清 扫术; 生存分析 